\title{
Social Agents Playing a Periodical Policy
}

\author{
Ann Nowé, Johan Parent, and Katja Verbeeck \\ Vrije Universiteit Brussel, Belgium \\ Computational Modeling Lab \\ asnowe@info.vub.ac.be, johan@info.vub.ac.be, kaverbee@vub.ac.be
}

\begin{abstract}
Coordination is an important issue in multiagent systems. Within the stochastic game framework this problem translates to policy learning in a joint action space. This technique however suffers some important drawbacks like the assumption of the existence of a unique Nash equilibrium and synchronicity, the need for central control, the cost of communication, etc. Moreover in general sum games it is not always clear which policies should be learned. Playing pure Nash equilibria is often unfair to at least one of the players, while playing a mixed strategy doesn't give any guarantee for coordination and usually results in a sub-optimal payoff for all agents. In this work we show the usefulness of periodical policies, which arise as a side effect of the fairness conditions used by the agents. We are interested in games which assume competition between the players, but where the overall performance can only be as good as the performance of the poorest player. Players are social distributed reinforcement learners, who have to learn to equalize their payoff. Our approach is illustrated on synchronous one-step games as well as on asynchronous job scheduling games.
\end{abstract}

\section{Introduction}

In multiagent systems the feedback experienced by an agent is usually influenced by the actions taken by the other agents present in the same system. Therefore modeling the other agents seems a natural thing to do. This leads to what is called the joint action space approach. As we will discuss below this approach suffers from some important drawbacks. On the other hand neglecting the presence of the other agents very often yields a suboptimal behavior of the global system. This is for instance the case in systems where resources are limited, such as job scheduling, routing and so on. In such systems agents should make agreements on how to share the resources to obtain a global optimal behavior, see [9]. Since communication has its price, we are interested in finding an approach where the communication between the agents can be kept low, yet yielding a fair solution to all agents. In this paper we present an approach inspired by the Homo egualis society described in sociology. The policy learned by the agents is a periodical policy, meaning that agents learn to play a repetitive combination of actions.

In many decision-making and strategy-settings people do not behave like the self-interested "rational" actors depicted in neoclassical economics and classical

L. De Raedt and P. Flach (Eds.): ECML 2001, LNAI 2167, pp. 382-393. 2001.

(C) Springer-Verlag Berlin Heidelberg 2001 
game theory [2]. In a Homo egualis society, individuals have an inequality aversion. As a result altruism appear in ultimatum and public good games. As Gintis states in 2] support for Homo egualis comes from the anthropological literature, describing how Homo sapiens evolved in small hunter-gatherer groups. Such societies had no centralized structure of governance, so the enforcement of norms depends on the voluntary participation of peers.

In section 5 we will show how the objective of equalizing the utility can be incorporated into distributed reinforcement learning agents with only limited communication.

The rest of the paper is organized as follows. In section 2 we discuss the join action space approach and why we believe this is not a solution to learning in multi agent systems. In section 3 we briefly look at the special case of dominance solvable games. Section 4 , deals with general sum games and why mixed policies are not the solution we are looking for. Section 5 introduces our approach of learning periodical policies, section 6 reports some experiments. Section 7 gives some ideas for future work and we conclude with section 8 .

\section{The Joint Action Space Approach}

For learning in a multiagent system, two extreme approaches can be recognized. On the one hand, the presence of other agents, who are possibly influencing the effects a single agent experiences, can be completely ignored. Thus a single agent is learning as if the other agents are not around.

On the other hand, the presence of other agents can be modeled explicitly. This results in a joint action space approach which recently received quite a lot of attention [15,6].

\subsection{What Is the Joint Action Space Approach}

In the joint action space technique, learning happens in the product space of the set of states $S$, and the collections of action sets $A_{1}, \ldots A_{n}$ (one set for every agent). The state transition function $T: S \times A_{1} \times \ldots \times A_{n} \rightarrow P(S)$ maps a state and an action from every agent onto a probability distribution on $S$ and each agent receives an associated reward, defined by the reward function $R_{i}: S \times A_{1} \times \ldots \times A_{n} \rightarrow P(\Re)$. This is the underlying model for the stochastic games, also referred to as Markov games in [5]6].

\subsection{Drawbacks of the Joint Action Space Approach}

The joint action space, is a safe technique in the sense that the influence of an agent on every other agent can be modeled. However the joint action space approach violates the basic principles of multi-agent systems : distributed control, asynchronous actions, incomplete information, cost of communication etc. 


\section{Learning Dominance Solvable Games}

For some classes of games, the joint action space approach is complete overkill. Any game which is dominance solvable1 can be tackled successfully by distributed, asynchronous reinforcement learning agents. A successful learning scheme in this situation is the reward-inaction scheme, where actions are selected probabilistically and actions which return high payoffs are positively reinforced, [8]. In fact what happens is an asynchronous, stochastic approximation of the well known iterated elimination of dominated strategies technique to obtain a Nash equilibrium strategy. In [7 a class of games which are called maximum games are proven to be dominance solvable. For an example see Figure 1 (left). However what happens when there isn't a Pareto dominant Nash equilibrium as in the Bach/Stravinsky game depicted in Figure 1 (middle). In the next section we discuss the use of a mixed policy for this case.

$$
\left(\begin{array}{ccc}
(9,11) & (5,6) & (1,0) \\
(5,6) & (5,6) & (5,6) \\
(1,0) & (5,6) & (1,0)
\end{array}\right),\left(\begin{array}{cc}
(2,1) & (0,0) \\
(0,0) & (1,2)
\end{array}\right),\left(\begin{array}{cc}
(10,1) & (0,0) \\
(0,0) & (1,2)
\end{array}\right)
$$

Fig. 1. Left: A maximum game of common interest. Middle: The Bach/Stravinsky game. Right: Variant of the Bach/Stravinsky game.

\section{Mixed Policies for General Sum Games}

An example of a game with more than one Nash equilibrium is the BachStravinsky game in Figure 1 (middle). There are two Nash equilibria $(2,1)$ and $(1,2)$. None of the Nash equilibria is Pareto dominant since they represent conflicting objectives for the two agents. If in this case the distributed, noncommunicating reinforcement learning approach should be applied, the strategy learned by the agents would converge to either of the Nash equilibria, see [8]. If the strategy to which the agents converge is the first one then agent 1 has his maximal possible payoff, and agent 2 is left with a suboptimal payoff, and visa versa for the other Nash equilibrium.

For the Bach-Stravinsky game, there is also a unique mixed Nash equilibrium, which is, agent one playing Bach with probability $2 / 3$ and Stravinsky with probability $1 / 3$, and agent 2 playing Bach with probability $1 / 3$ and Stravinsky with probability $2 / 3$. The question is however, why should we like agents to learn this mixed strategy? If we compute the expected payoffs both agents receive in

\footnotetext{
${ }^{1}$ A strategic game is dominance solvable if all players are indifferent to all outcomes that survive the iterative procedure in which all the weakly dominated actions of each player are eliminated, see 11 .
} 
this case, we get a payoff of $2 / 3$ for both agents, so both get less than what they get in the pure Nash equilibria. So on the one hand mixed strategies are not necessarily better than pure strategies, on the other hand playing pure Nash equilibria is often unfair to one of the agents. This brings us to the next section where we introduce the notion of a periodical policy.

\section{$5 \quad$ Learning Fair Periodical Policies}

As already mentioned in the introduction the idea of learning periodical policies is inspired by the Homo egualis society from sociology. In a Homo egualis system, agents do not only care about their own payoff, but also about how it compares to the payoff of others. A Homo egualis agent may be willing to reduce his own payoff to increase the degree of equality in the group. On the other hand he is also displeased when receiving a lower payoff than the other group members. A Homo egualis society can be modeled following [3] where the utility function of player $i, u_{i}$ in an $n$-player game is:

$$
u_{i}(x)=x_{i}-\frac{\alpha_{i}}{n-1} \Sigma_{x_{j}>x_{i}}\left(x_{j}-x_{i}\right)-\frac{\beta_{i}}{n-1} \Sigma_{x_{j}<x_{i}}\left(x_{i}-x_{j}\right)
$$

Where $x=\left(x_{i}, \ldots, x_{j}\right)$ are the cumulative payoffs for each player respectively and $0 \leq \beta_{i}<\alpha_{i} \leq 1 . \beta_{i}<\alpha_{i}$ reflects the fact that Homo egualis exhibits a weak urge to inequality when doing better than the others and a strong urge to reduce reduce inequality when doing worse than the others.

This idea can be translated into distributed reinforcement learning agents with limited communication. First we concentrate on the second term in formula (1), i.e. the term expressing that agents with a higher payoff than other group members are willing to share some of their payoff with the less fortunate agents. This is realized as follows in the learning algorithm. Agents are distributed Q-learners who learn a stateless action function which is updated every game iteration 2 with the immediate payoff they receive in the previous game. Without communication they start exploring their action space and as the agents are selfish utility optimizing reinforcement learners, the group will converge to a Nash equilibrium, 8]. After a given period of time a communication phase will take place in which all agents send their cumulative payoff as well as their average payoff collected in the last non-communication period, to each other. The agent who experiences the highest cumulative payoff and the highest average payoff in the last period will exclude the action he is currently playing so as to give the other agents the opportunity to converge to a more rewarding situation. In more formal terms, after convergence to one of the Nash equilibria, the hyperplane containing the action of the best performing agent is removed from the joint action space, and a new learning period is stared in which the resulting subspace is explored. During this new exploration phase there is again

\footnotetext{
${ }^{2}$ One time unit corresponds to one game iteration.
} 
no communication between the agents. After a while the group will converge to another Nash equilibrium and one of the other agents might have caught up with the former best agent. Again communication takes place and the currently "best" agent can be identified. If this is still the previous best agent nothing will happen 3 . However if another agent has outperformed the previous best one, the latter will release again his excluded action. As a result another subspace is explored, and the group will seek for yet another Nash equilibrium. As a result agents play a periodical policy, they alternate between the different Nash equilibria of the game. Even in games were the different Nash equilibria do not give the same best payoff as in Figure 1] (right) the algorithm is able to alternate between the Nash equilibria such that the average payoff for all players is equalized 4 . In Figure 2 the above described algorithm for learning fair periodical policies, is given in pseudo code.

\section{Some Experiments}

In this section we test the previous described algorithm on both synchronous and asynchronous games. Some preliminary results were already reported in [10]. To test the scalability of our approach in more complex action spaces, we also experimented with 3 to 5 player games with action spaces of 3 and 5 actions respectively.

The representatives of the synchronous type of games we tested are the Bach/Stravinsky game of Figure 1 (middle) and a variant shown in Figure 1 (right). In the variant game the Nash equilibrium $(1,1)$ gives 5 times more payoff to player 1 , than the Nash equilibrium $(2,2)$ gives to player 2.

The asynchronous games we played are asynchronous network games of the common pool resource type [4] which were simulated in the QNAP2 network modeling language 14. The simplest game being the job scheduling game depicted in Figure 3. This job scheduling game has two pure Nash equilibria: one player always chooses the common resource and the other one uses the private resource and vice versa. Note that the payoff should be minimized here. Although the common resource looks more interesting to use from an agents' point of view (because it can handle the jobs more quickly on average), the benefit disappears when there is an overconsumption of the common good by all the agents.

\subsection{Results on Symmetric Games}

When the players use a Q-learning algorithm [13, they find in both games one of the Nash equilibria as is shown in the payoff each players receives, see Figure 4

3 The agent needs the average payoff over the previous period to determine whether he was playing his best Nash equilibrium in the last period or if someone else was trying to catch up, but actually needs more time to do so. In this case the social agent will keep his excluded action excluded.

4 This because the average payoff of the last period is also communicated. This prevents a player to free an excluded action when another agent who is currently playing his best Nash equilibrium needs more time to catch up. 


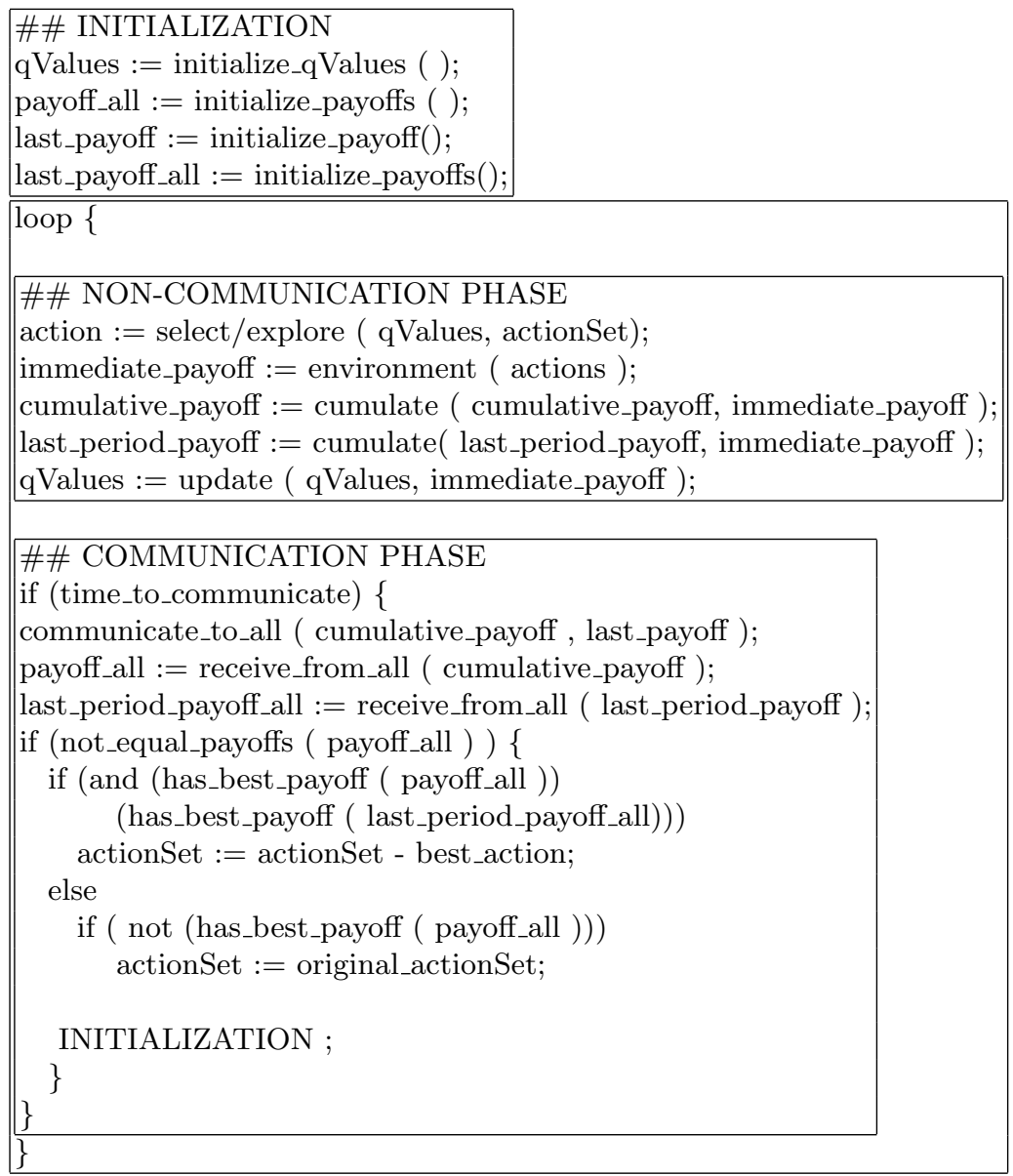

Fig. 2. Pseudo code of the algorithm executed by each Homo egualis Q-learning player.

(top). The left column gives the result for the Bach Stravinsky game, the right column gives the results for the variant of the Bach/Stravinsky game. One of the players seems to win the game, while the other one performs sub-optimally. In both cases the learning rate $\alpha$ is 0.1 .

When the Q-learning players use a Homo egualis argument, by setting up a communication channel between them, a periodic policy is learned which alternates between the different pure Nash equilibria of the game. See Figure 4 (left-middle) and Figure 4 (right-middle) for the equalization of the payoff. The average payoff to which they converge goes to the optimal, which is 1.5 for the standard Bach/Stravinsky game and 1.9 for the variant. For the Bach/Stravinsky game this proofs that both equilibria are played equally. While in the variant of this game, the Nash equilibrium $(2,2)$ is played 9 times for every period 
A1

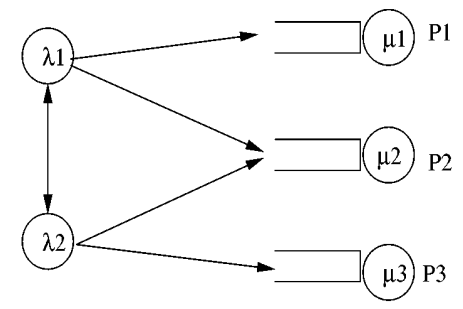

Fig. 3. A simple job scheduling game. Two agents can use either their private processor or a common processor. The agents generate jobs according to an exponential law with a mean value of 1.5 time units. Both the private processors handle the jobs with a time consumption chosen from an exponential distribution with mean value of 1 time unit, while the common processor works with a mean of 0.7 time units.

of play of the other Nash equilibrium $(1,1)$. Figure 4 (right-middle)shows that the period in which player 1 climbs is $1 / 9$ of the period in which it drops. In the Bach/Stravinsky game communication was done every 50 time units, in the variant of the game communication size was every 100 time units.

Finally Figure 4 (left-bottom) and Figure 4 (right-bottom) gives the payoffs of the players when both players play their mixed Nash equilibrium strategy for the two symmetric games respectively. For both games the average payoff isn't as good as for the periodical policy, and in the variant of the Bach/Stravinsky game the payoffs aren't equalized at all.

\subsection{Results on Asymmetric Games}

In the asymmetric games, agents keep generating jobs independently of one another and feedback from the processors only arrives at certain time intervals. So the action selection isn't synchronous anymore as in the previous games and reinforcement is delayed. Using synchronous communication and social rules as described by the Homo equalis condition, periodical policies equalizing the job turn around time can still be found.

Figure 5 shows the payoff for the agents in the job scheduling game of Figure 3 for respectively 2 Q-learners, 2 Homo egualis Q learners and 2 players playing their mixed Nash equilibrium. As in the synchronous games, 2 Q-learners converge to one of the Nash equilibria, meaning here one player can use the common processor while the other has to use his own. Two Homo egualis players however alternate between using the common and private processor separately. Remarkable to note is that while playing the periodic policy the players implicitly learn the policy for playing the mixed Nash equilibria off-line. When the mixed Nash equilibrium is played the payoff also has the tendency to be equalized, however the average payoff isn't that good and in real-world situations where jobs may be relatively large, the discrete game may be approximated and the periodic policy may give more security for the players. Communication between 2 Homo egualis players is done every 2000 time units. 

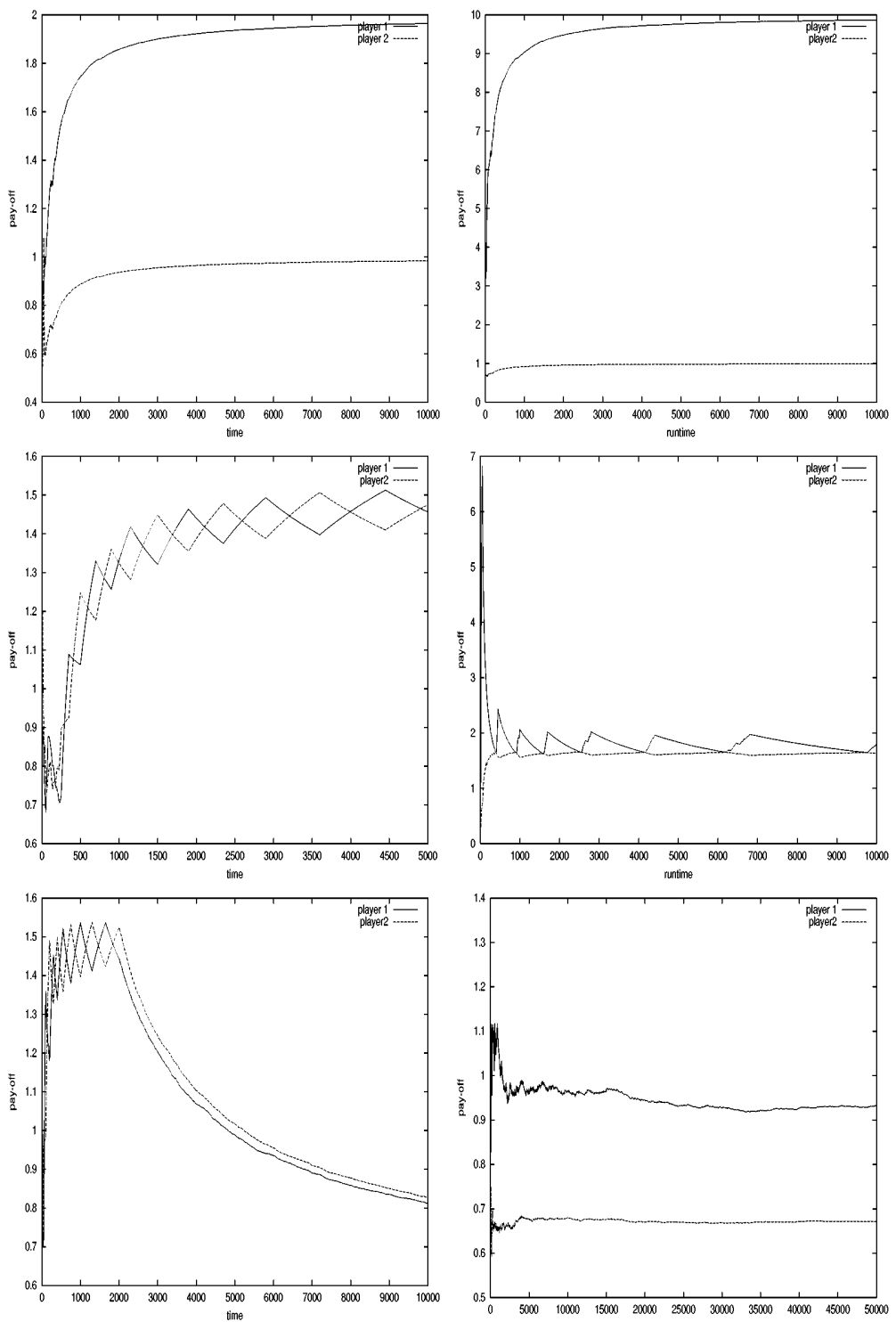

Fig. 4. Left:Bach/Stravinsky game. Top: the average payoff for 2 Q-learners, Middle: the average payoff for 2 Homo egualis Q-learners, Bottom: the average payoff for 2 players playing the mixed Nash equilibrium.

Right: Variant of the Bach/Stravinsky game. Top: the average payoff for 2 Q-learners, Middle: the average payoff for 2 Homo egualis Q-learners, Bottom: the average payoff for 2 players playing the mixed Nash equilibrium 

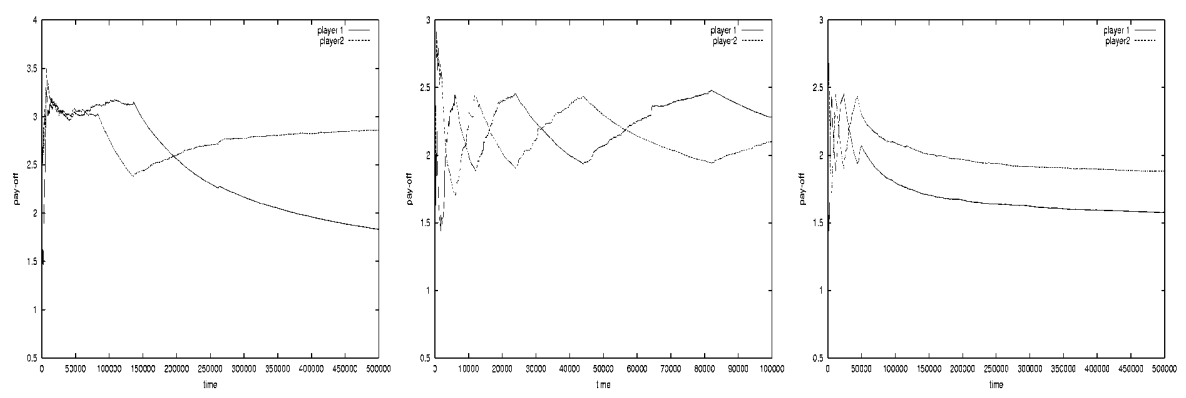

Fig. 5. Job scheduling game. Left: the average payoff for 2 Q-learners, Right: the average payoff for 2 Homo egualis Q-learners, Bottom: the average payoff for 2 players playing the mixed Nash equilibrium.

\subsection{Results in More Complex Action Spaces}

Our algorithm was also tested on games with more than two players and games in which each player has more than two actions. Figure 6 (left, up) shows the evolution of the payoff for an extension of the Bach/Stravinsky game to what we call the Bach/Stravinsky/Mozart game. There are now three Nash equilibria with payoff outcomes $(2,1,1),(1,2,1)$ and $(1,1,2)$ for respectively the joint actions $(1,1,1),(2,2,2)$ and $(3,3,3)$. In the same way we can extend this game to 5 players each having 5 actions and each preferring another equilibrium of the 5 available Nash equilibria. Figure 6 (left, bottom) gives the results for this further extended game. Communication is done every 100 time steps.

We also tested an extension of the job scheduling game of Figure 3 . In this game there is a third agent sending jobs to either his private processor or the common one. The third agent generates jobs with the same load as the other ones and the private processor works as fast as the other private processors. The results of the synchronous simulation of this game and a further extended game to 5 players is found in Figure 6 (right). Communication here is done every 300 time steps.

In all these extensions, the action spaces are more complex than in the previous two player games, yet our algorithm still manages to equalize the payoffs. However these extensions reveal the importance of the length of the noncommunication period. When the action space is more complex, it takes more time for the agents to coordinate and converge to a Nash equilibrium.

\section{Future Work}

At this point the Homo equalis player is implemented so that only when an agent receives a higher payoff than the other group members an effort is made to share some of their payoff with the less fortunate agents. The Homo equalis player described in [2] also has a strong urge to equalize the payoffs when being 

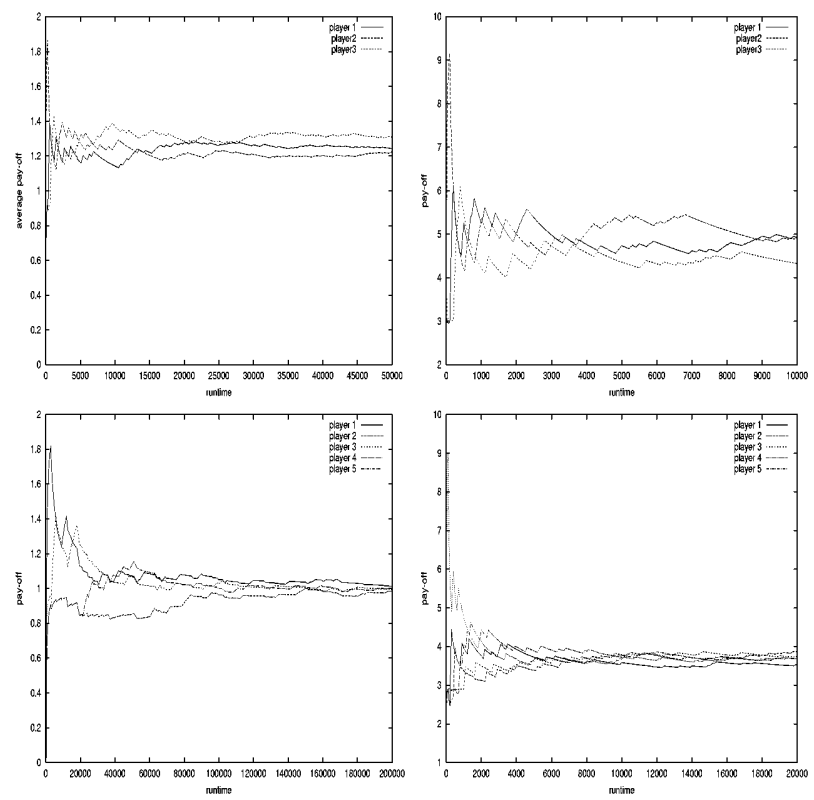

Fig. 6. Left: The average payoff for 3 (up) / 5 (bottom) Homo equalis players in the extended Bach/Stravinsky game. Right: The average payoff for 3 (up) / 5 (bottom) Homo-equalis players in a synchronous version of the Job Scheduling game with 3 / 5 agents, $3 / 5$ private processors and one common processor. A payoff of 10 is collected when using the common processor alone, while a payoff of 3 is collected while using the private processor.

on the bottom. This is reflected in the third term of equation (1). This term can be incorporated in the learning system by increasing the learning rate of agents who have a low accumulated utility compared to the others. This will give these poor agents a higher probability to pull the group towards their favorite Nash equilibrium. This has not been implemented yet, but we believe that this idea might be a solution to games where some Nash equilibria are found faster than others. Especially when concerned with games in complex action spaces and multiple players, convergence to a Nash equilibrium may take a while. Exploration should be done carefully and the non-communication part should be long enough for the equilibrium to be found and played. Here also the adaptation of the learning rate of the agents might speed up convergence. Maybe after a while the agent can learn the sequence of Nash equilibria to play.

We also assumed that the games we played had some pure Nash equilibria with conflicting concerns. Of course when this is not the case and a player feels that even when he is forced to a new Nash equilibrium which still favors the previous best player, a periodical policy is not the answer. 


\section{Conclusion}

This work studied the fairness of strategies learned by agents in a game theoretic setting, whereby the game assumes competition between the players, but the overall performance can only be as good as that of the poorest player. The global goal is thus to equalize the payoffs of the players as well as possible. The chosen test cases are synchronous games and an asynchronous common good job scheduling game with pure Nash equilibria and a mixed Nash equilibrium. The pure equilibria are not fair in equalizing the payoff. The mixed equilibrium was shown not to be interesting in the synchronous game and not practical in the asynchronous game.

The solution proposed is for agents to learn a periodic policy in a Homo egualis society. Communication is needed only to compare performances and to reduce the action spaces to go find alternative Nash equilibria. In case the pure Nash equilibria don't give the same payoff to all the players, our algorithm was able to learn the length of the period each agent needs to equalize the average payoff. As a side-effect the mixed Nash strategy is learned off-line while playing the period policy.

To conclude, in evolutionary terms, the difference between a periodic policy and a mixed one can be described by the difference between a mixed population of entities playing pure strategies and a population of entities playing mixed strategies, see [12].

\section{References}

1. Claus C., Boutilier C.,: The dynamics of reinforcement learning in cooperative multi-agent systems. Proceedings of the fifteenth National Conference on Artificial Intelligence,(1998) p 746 - 752.

2. Gintis H.,: Game Theory Evolving: A Problem-Centered Introduction to Modeling Strategic Behavior. Princeton University Press, 2000.

3. Fehr E., Schmidt K.M.,: A Theory of Fairness, Competition, and Cooperation. Quarterly Journal of Economics, 114 (1999) p 817 - 868.

4. Hardin G.,: The tragedy of the commons. Science 162 (1968) p1243-1248.

5. Hu J., Wellman M. P.,: Multi Agent Reinforcement Learning in Stochastic Games. Submitted, 1999.

6. Litmann M.L.,: Markov games as a framework for multi-agent reinforcement learning. Proceedings of the Eleventh International Conference on Machine Learning, (1994) p $157-163$.

7. Mariotti M.,: Maximum Games, Dominance Solvability, and Coordination. Games and Economic Behavior 31, (2000) p 97 - 105.

8. Narendra K., Thathachar M., : Learning Automata: An Introduction. Prentice-Hall (1989).

9. Nowé, A., Verbeeck, K.,: Distributed Reinforcement learning, Loadbased Routing a case study. Proceedings of the Neural, Symbolic and Reinforcement Methods for sequence Learning Workshop at ijcai99.

10. Nowé, A., Verbeeck, K., Lenaerts T.,: Learning Agents in a Homo Egualis Society Technical report Computational Modeling Lab. Maart 2001. Vrije Uniuversiteit Brussel (2001). 
11. Osborne J.O.,Rubinstein A., :A course in game theory. Cambridge, MA: MIT Press (1994).

12. Smith J.M.,: Evolution and the Theory of Games. Cambridge University Press (1982).

13. Sutton, R.S., Barto, A.G. : Reinforcement Learning: An introduction. Cambridge, MA: MIT Press (1998).

14. QNAP2 reference manual, SIMULOG 1996. 